REVIEW ARTICLE

\author{
J.B. White \\ C.G.M. Ken \\ H.J. Cloft \\ D.F. Kallmes
}

\section{Coils in a Nutshell: A Review of Coil Physical Properties}

\begin{abstract}
SUMMARY: Since its inception, endovascular coil technology has grown substantially as multiple manufacturers entered the market with an ever-increasing number of new products. Practitioners are now inundated with a choice of coils that vary on the basis of factors such as size, composition, stiffness, and detachment mechanism. The seasoned interventionalist had the benefit of evolving with this technology and, therefore, is likely to understand many of the practical nuances of coil development; for more junior practitioners, who did not experience the ongoing changes in technology, this review will provide a basic framework for the fundamentals of coil design.
\end{abstract}

$\mathbf{0}$ $\mathrm{f}$ all the coil properties that are considered before fabrication, biocompatibility is the most important. A biocompatible coil is one that is composed of primarily inert material that allows an effective treatment without the concern for a systemic host response. Metal alloys with a proved record for patient safety have been the main sources for coil production. Nitinol, platinum, nickel, iridium, and tungsten have been the primary metals used in construction and are usually developed as alloys to reach an optimal strength. Metal strength is determined experimentally and is referred to as the modulus of rigidity or shear modulus $(G)$. The modulus of rigidity is the coefficient of elasticity for a shearing force, defined as the ratio of the shear stress to the shear strain. Modulus of rigidity can be experimentally determined from the slope of a stress-strain curve created during torsion tests conducted on a metal sample. The relative strength of the metals and their alloys frequently used in coil manufacturing is shown in Fig 1. A platinum (92\%)/tungsten (8\%) alloy has become the mainstay material for most current coil designs. All of these metals in pure and alloy form are readily available in hundreds of permutations from distributors worldwide (Appendix A).

\section{Configuration}

Once a metal is chosen, the coil is created by undergoing a series of transformations from a primary $\left(1^{\circ}\right)$ to secondary $\left(2^{\circ}\right)$ to tertiary $\left(3^{\circ}\right)$ structure (Fig 2$)$. The primary structure is the "stock" wire, which is fabricated in linear form with a diameter $\left(D_{1}\right)$ of any range. Most stock wires used for coil manufacturing range from 0.00175 to 0.003 inch. The stock wire diameter, $\mathrm{D}_{1}$, is the central factor in determining coil "stiffness," which is discussed in more detail below.

The stock wire is wound around a mandrel, also of varying diameter, to produce the secondary structure of the coil (Fig $2)$. The diameter $\left(D_{2}\right)$ of the secondary structure, in conjunction with the number of turns per unit of length around the mandrel, represents 2 additional factors that impact product stiffness. The secondary diameter, $\mathrm{D}_{2}$, dictates the historic coil grouping, in which coils deemed "T10" coils are typically

Received October 30, 2007; accepted October 31.

From the Departments of Neurological Surgery (J.B.W.) and Radiology (H.J.C., D.F.K.), Mayo Clinic, Rochester, Minn; and independent coil engineer (C.G.M.K.).

Dr. Kallmes receives research support from Micrus and MicroVention. Dr. Cloft receives research support from Cordis.

Please address correspondence to David F Kallmes, MD, Mayo Clinic, Department of Radiology, 200 First St SW, Rochester, MN 55905; e-mail: kallmes.david@mayo.edu

DOI 10.3174/ajnr.A1067 wound to approximately 0.010 inch and coils deemed "T18" coils are typically wound to approximately 0.015 inch. However, many manufacturers now produce coils with $\mathrm{D}_{2}$, at several steps between 0.010 and 0.015 inch, including 0.012 -inch (Cordis, Miami Lakes, Fla) and 0.014-inch (Micrus Endovascular, San Jose, Calif) coil lines. $\mathrm{D}_{2}$ has important implications for both stiffness and packing attenuation.

Finally, the secondary structure can be shaped into any number of tertiary configurations (helical, complex, spherical, etc), which also are developed with a specific diameter $\left(D_{3}\right)$ and length (L), parameters that serve as a central factor in package labeling and coil selection during interventional procedures. For instance, coils are typically packaged as " $3 \mathrm{~mm} \times$ $4 \mathrm{~cm}$," where the millimeter measurement is that of the tertiary diameter, $D_{3}$, and the centimeter measurement is that of L. Just as the metals for coils are readily available, so too are the fabrication companies that are capable of shaping metals into an endless number of designs (Appendix A).

\section{Stiffness}

Coil stiffness, or more aptly as it is marketed as its inverse, softness, has become one of the fundamental tenets of coil selection by practitioners. This has largely stemmed from the marketing bonanza of manufacturers providing alternatives to the "hard" coils in the form of "soft," "supersoft," and "ultrasoft" coils. Without question, the ability to place a coil in an aneurysm will be enhanced by the softness of the coil, so understanding softness and stiffness on a theoretic basis may aid the practitioner in choosing specific coils.

To understand coil stiffness, one needs to reference the physical properties of springs. The stiffness of a spring is directly proportional to its spring constant $(\mathrm{k})$. The same relationship is true for coils as they take their secondary shape:

$$
k=\frac{D_{1}^{4} G}{8 D_{2}^{3} n}=\text { Stiffness } \propto \frac{D_{1} G}{D_{2} n} .
$$

Another way to put this relationship is the smaller the value of $k$, the softer the coil. Accordingly, stiffness is directly proportional to $D_{1}$ to the fourth power and linearly related to the $G$ of the stock wire and inversely proportional to the cube of the mean $\mathrm{D}_{2}$ of the coil. Reflecting on those parameters for just a moment here, and in more detail in the following paragraphs, one can see that anything raised to the fourth power, or even the third power, must be very important. The stiffness is inversely related to the number of wraps per unit distance (n) made on the shaping mandrel when forming the secondary 


\section{Relative Metal Hardness}

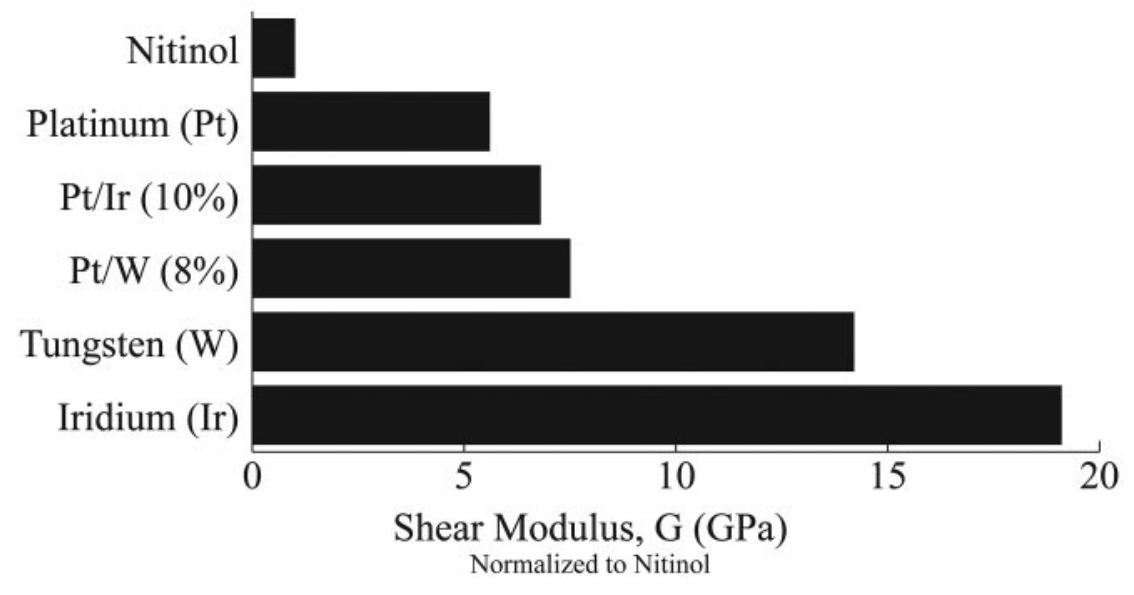

Fig 1. The relative hardness of the metals and their alloys commonly used to construct coils. The $\mathrm{G}$ of each metal and alloy is referenced and then normalized to a nitinol value of 1 gigapascal (GPa).

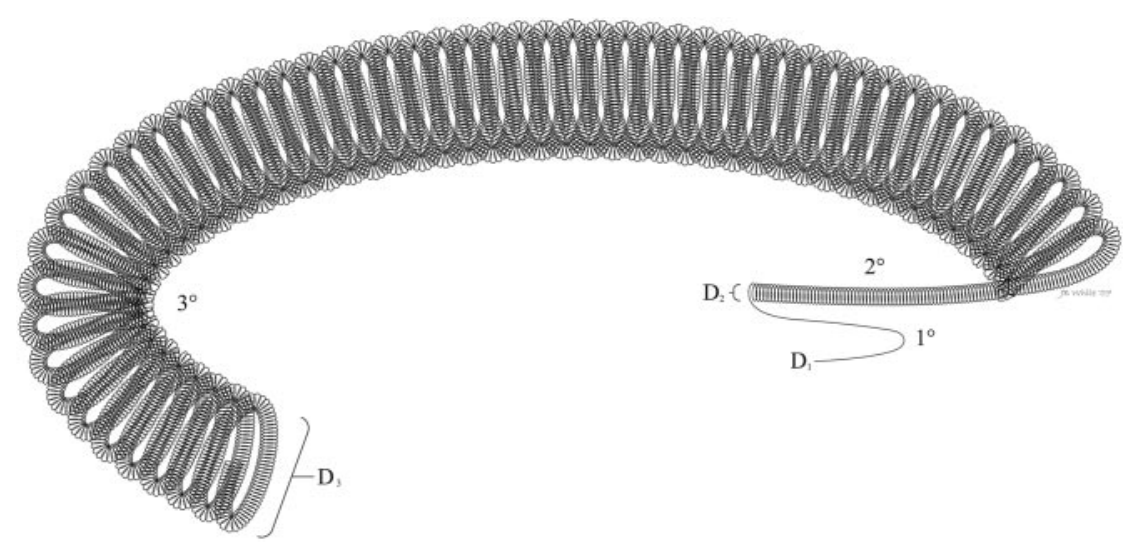

Fig 2. A cartoon depicting a helical coil design with representations of the primary $\left(1^{\circ}\right)$ configuration with $D_{1}$, the secondary $\left(2^{\circ}\right)$ configuration with $D_{2}$, and the tertiary $\left(3^{\circ}\right)$ configuration with $\mathrm{D}_{3}$. configuration, also known as the primary wind of the coil. For example, a greater number of turns (larger $n$ ) are achieved when the stock wire is wrapped tightly. A more tightly wound coil results in a smaller space between coil loops; this interloop space is known as the "pitch." The pitch of a coil is often adjusted by manufacturer to fine-tune softness or allow penetration of expansive materials within the coil lumen. Pitch is usually notated as a fraction (ie, 10\%) of the stock wire diameter and is proprietary information to each company. The spring equation does not account for nonmetal coatings or fillings added to coils, such as expandable polymers or suture, which may have a substantial impact on coil stiffness.

From a practical standpoint, $\mathrm{D}_{1}$ is the dominant feature that predicts stiffness. First, its impact on stiffness is as a fourth power, compared with a third power for $\mathrm{D}_{2}$. Second, most coils are made from similar alloys, thus with similar $\mathrm{G}$ and little or no pitch and thus similar $\mathrm{n}$. Stock wire diameters typically range from 0.00175 to 0.003 inch, with the former diameters used in softer smaller coils and the latter used in larger stiffer coils. Note that on the basis of the value for $\mathrm{D}_{1}$ alone, the relative stiffness between the smallest $(0.00175 \mathrm{inch})$ and largest $(0.003 \mathrm{inch}) \mathrm{D}_{1}$, is predicted to be $9: 1$. In other words, all else being equal, a coil manufactured from 0.003 -inch stock wire would be $>800 \%$ stiffer than one constructed from 0.00175 -inch stock wire. It is, therefore, no surprise that smaller coils constructed from 0.00175 -inch stock wire, such as a Cashmere coil (Micrus Endovascular), are markedly easier to pack into an aneurysm compared with a larger coil constructed from 0.003-inch stock wire, such as a standard Orbit Trufill coil (Cordis).
To expand market choices, manufacturers will produce coils of the same $\mathrm{D}_{2}$ but will make micron-sized changes in $\mathrm{D}_{1}$. For example, a 10-coil, depending on the desired softness, may be created with stock wire diameters of $0.00150,0.00175$, or 0.002 inch. Although the difference among these wires represents only 0.00025 inch, the change in stiffness is exponential. Thus, the relative stiffness of a soft coil made from 0.002 inch compared with that made from 0.0015 -inch wire would be $3: 1$. A simplified ratio of the spring constant equation $\left(\mathrm{D}_{1} / \mathrm{D}_{2}\right)$ can be derived for each coil to compare softness between coil lines (Fig 3). Stock wire of varying diameters can be purchased by the spool or specified when coils are ordered for fabrication.

The $\mathrm{D}_{2}$ also significantly impacts both coil softness and packing attenuation. Stiffness is inversely proportional to the cube of $\mathrm{D}_{2}$. Thus, a stock wire wound to 0.012 inch will have a stiffness value of only $57 \%$ of the same stock wire wound to 0.010 inch. Further, the 0.012 -inch device will offer $44 \%$ more packing attenuation compared with the 0.010 -inch device (details on how $\mathrm{D}_{2}$ impacts packing attenuation are offered below). In other words, the wire wound to a larger $\mathrm{D}_{2}$ will not only offer much greater filling volume but will also be substantially softer. A plot of coilfilling volume versus softness for the coils shown in Fig 3 is presented in Fig 4 to demonstrate this relationship.

The $\mathrm{D}_{3}$ of the coil (helical, complex, etc) also impacts coil stiffness. A coil made with identical $\mathrm{D}_{1}$ and $\mathrm{D}_{2}$ but a larger $\mathrm{D}_{3}$ will be softer. This is observed by plugging the $\mathrm{D}_{2}$ as $\mathrm{D}_{1}$ into the spring equation and substituting $\mathrm{D}_{3}$ for $\mathrm{D}_{2}$. Some companies have worked to control this effect by increasing the stiffness of the secondary configuration as tertiary diameters become larger. 


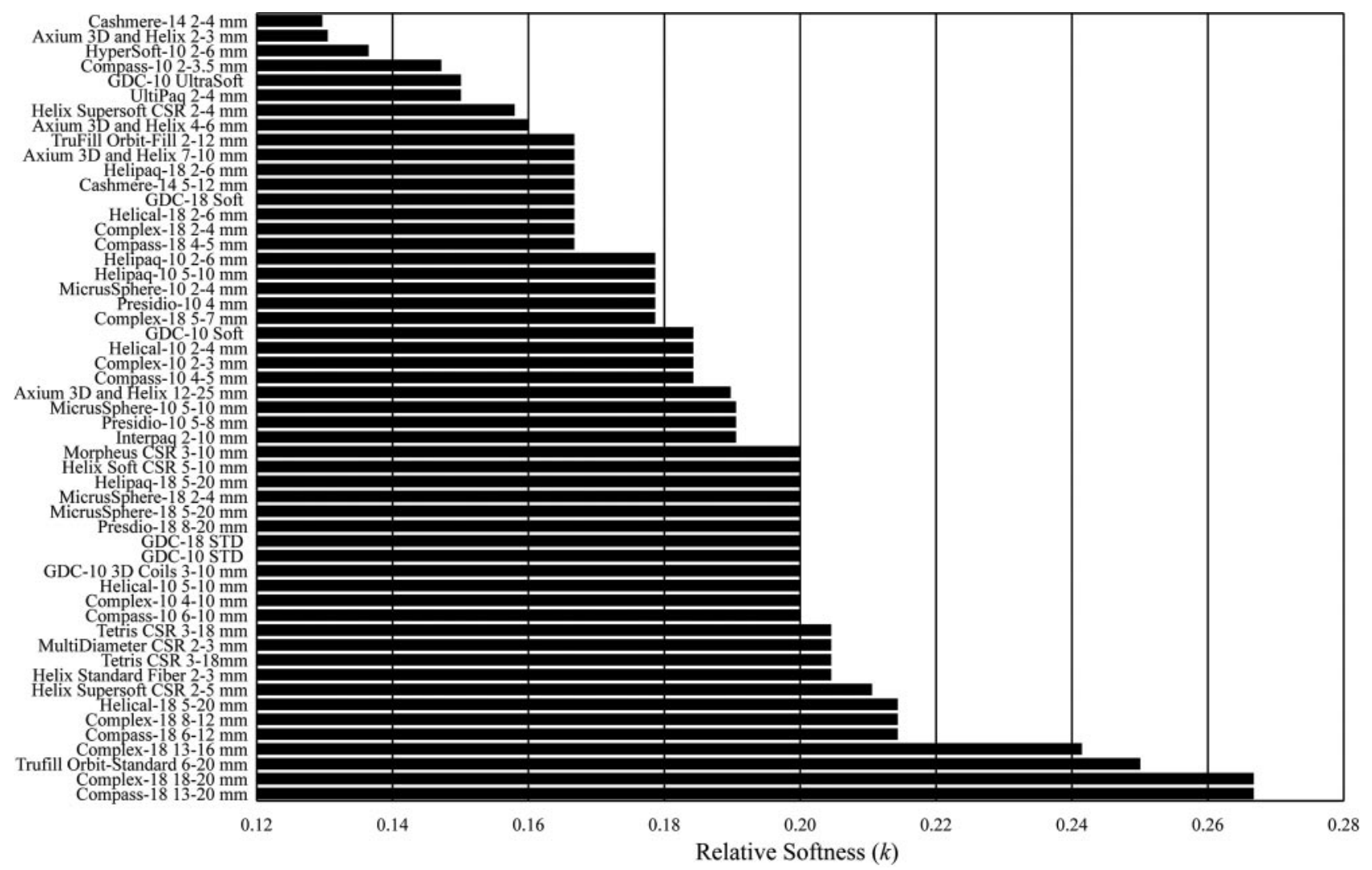

Fig 3. The relative coil softness for numerous coil lines developed from the 5 companies that manufacture them. For each coil, a simplified spring-constant equation $\left(D_{1} / D_{2}\right)$ was used to derive $k$. This simplified equation makes several assumptions, including an identical value for $\mathrm{G}$ and $\mathrm{n}$. It also negates the contribution of various stretch-resistant and bioactive components. The $y$-axis shows the name of each coil line. The $x$-axis reveals the $k$ for each coil. The smaller the value of $k$, the softer the coil.

Given that some of the variables in the spring constant equation are held tightly by manufacturers, the reverse engineering of coils requires obtaining a direct measurement of stiffness by solving Hooke's law $(\mathrm{F}=k \mathrm{x})$ for a given coil. Manufacturers' brochures showing comparisons between their coil products and others often emphasize softness as determined by bench-top testing. A Hooke's law apparatus sensitive to minute changes in length is used to derive the spring constant. Once this information is obtained, other variables $\left(D_{1}, D_{2}\right.$, and $\left.G\right)$ that can be obtained by direct measurement allow the calculation (equation 1) of those variables (n), which are more difficult to measure. An industry standard to experimentally determine stiffness by using bench-top testing does not exist.

\section{Volume}

The packing attenuation of aneurysms with coils is thought to be related inversely to the rate and degree of aneurysm recurrence. Studies have shown that the average packing attenuation of coiled aneurysms can range from $20 \%$ to $73 \%$, depending on the type of coil used and how aggressively one packs the aneurysm. ${ }^{1-5}$ These numbers are determined by calculating the total volume of coils placed within the aneurysm and dividing by the volume of the aneurysm coiled. Coil volume is proportional to $\mathrm{L}$ and $\mathrm{D}_{2}$ of the coil (equation 2). Aneurysm volume is normally determined by 1 of 2 methods, both of which have been validated and carry an acceptable margin of error when done correctly. ${ }^{6,7}$ The first method uses volumetric equations for known geometries (Appendix B) that approxi- mate the aneurysm shape. The aneurysm coordinates are measured from either $2 \mathrm{D}$ or $3 \mathrm{D}$ images. The second method derives the aneurysm volume from 3D-rendering software, which allows the user to conform a graphic to the aneurysm, which then calculates the volume on the basis of the number of voxels within the graphic.

$$
V=L \times \text { Area }=L \times \frac{\pi D_{2}}{4} .
$$

In addition to coil volume, manufacturers have argued that coil stiffness and configuration (tertiary) also serve as important factors in increasing aneurysm packing attenuation while reducing the likelihood for coil compaction. Although some of these claims may seem intuitive, proof that a soft complex coil is superior to a standard helical coil in terms of aneurysm packing and clinical outcome has not been definitively demonstrated.

\section{Conclusions}

It has been more than a decade since the first detachable coils were introduced. Although there has been a steady stream of changes in coil technology, the basic characteristics of coil design have remained, for the most part, the same. Undoubtedly, new materials will be incorporated into the design of coils or will altogether replace them. A basic understanding of this technology as it progresses will allow the consumer to make educated and patient-safe decisions. 

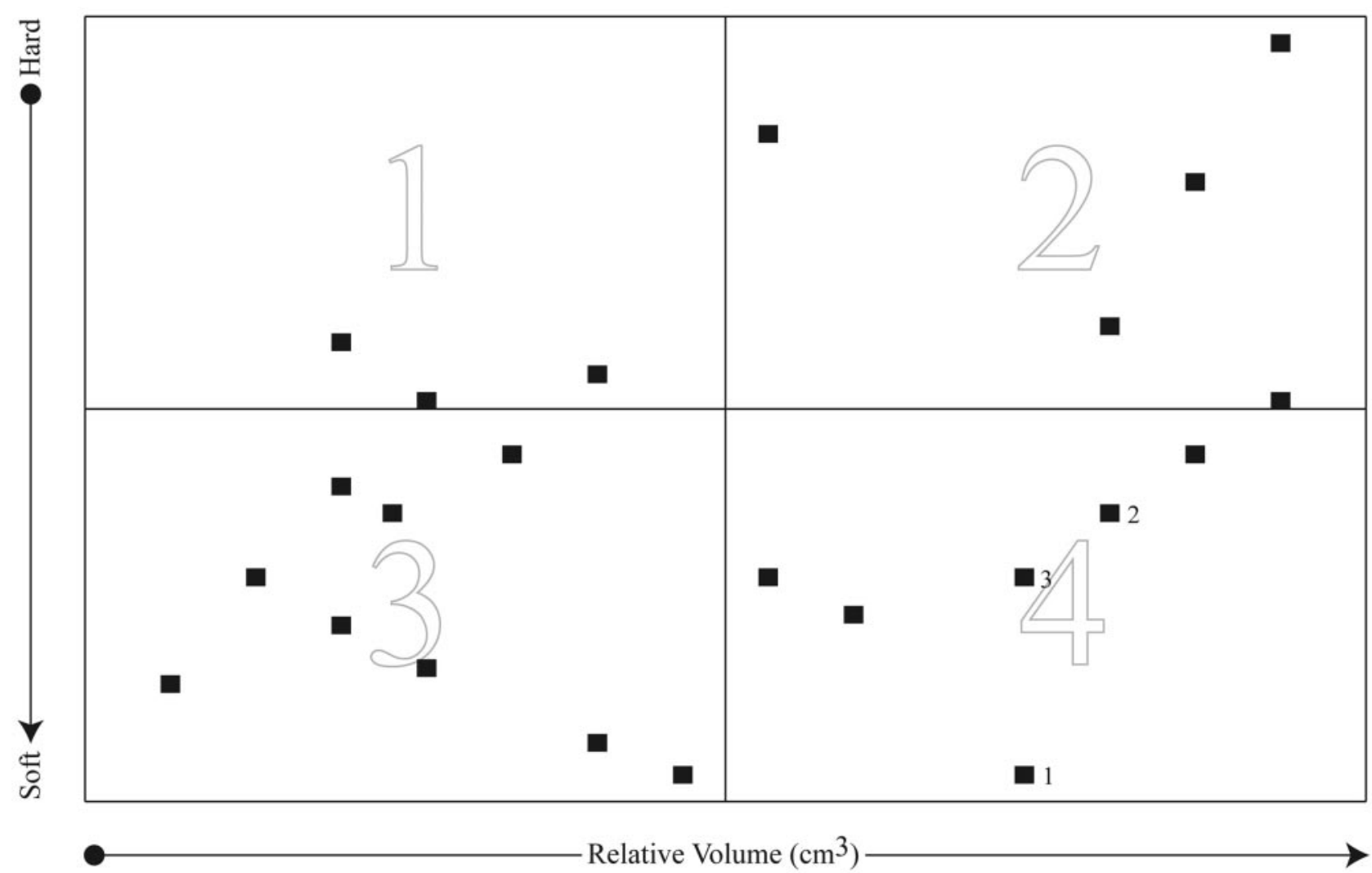

Fig 4. A graph that compares the softness (y-axis) of each coil as it relates to its filling volume (x-axis). Softness is determined as in Fig 3 . The volume for each coil is shown as a function of $D_{2}$, which assumes an identical length for all coils compared. Softer coils are lower on the $y$-axis. Coils with a greater filling volume fall farther along the $x$-axis. The graph is divided into 4 quadrants as the watermarks illustrate. Quadrant 4 encompasses coils that are the softest while maintaining greater filling volumes. Conversely, quadrant 1 contains the "stiffest" coils with the lowest filling volume. The numbered squares in quadrant 4 represent the 6 softest coils with the greatest filling volume. The total number of squares in this figure is smaller than the number of coils compared due to identical overlap of some coils. $1=$ Cashmere $14(2-4 \mathrm{~mm}) ; 2=$ Complex 18 (5-7 mm); and $3=$ Axium 3D and Helix (7-10 mm), Helipaq-18 (2-6 mm), Cashmere 14 (5-12 mm), GDC-18 Soft, Helical 18 (2-6 mm), Complex 18 (2-4 mm), and Compass 18 (4-5 mm).

\section{Appendix A}

The authors present the following companies as references and have no disclosures or conflicts of interest related to them:

\section{Wire Manufacturers}

Johnson Matthey (London, UK)

Sigmund Cohn (Mount Vernon, NY)

Heraeus (Hanau, Germany)

\section{Coil Winding/Fabrication Companies}

Heraeus Vadnais (St. Paul, Minn)

Motion Dynamics (Fruitport, Mich)

Accellent (Wilmington, Mass)

\section{Appendix B}

The following volumetric equations may be used to estimate the volume of an aneurysm. The shape of the aneurysm determines the equation that is used. These equations become the denominator of the ratio used to determine coil-packing attenuation. The nominator is equation 2 .

Cylinder: $\nu=\pi r^{2} \times h$

Sphere: $\nu=\frac{4}{3} \pi r^{3}$

Ellipsoid: $\nu=\frac{4}{3} \pi r_{1} r_{2} r_{3}$

\section{Appendix C}

The following is a list of coils and their respective manufacturers used for analysis in this manuscript. At the request of each company, the specific diameters of each coil configuration was not revealed. Instead, ratios were devised as described in the text for the purposes of comparison.

\begin{tabular}{llr}
\hline Company & \multicolumn{1}{c}{ Type } & \multicolumn{1}{c}{ D $_{3}$} \\
\hline Cordis & Standard & $6-20 \mathrm{~mm}$ \\
Cordis & Fill & $2-12 \mathrm{~mm}$ \\
ev3 & AXIUM 3D and AXIUM Helix & $2-3 \mathrm{~mm}$ \\
ev3 & AXIUM 3D and AXIUM Helix & $4-6 \mathrm{~mm}$ \\
ev3 & AXIUM 3D and AXIUM Helix & $7-10 \mathrm{~mm}$ \\
ev3 & AXIUM 3D and AXIUM Helix & $12-25 \mathrm{~mm}$ \\
ev3 & Morpheus CSR & $3-10 \mathrm{~mm}$ \\
ev3 & Tetris CSR & $3-18 \mathrm{~mm}$ \\
ev3 & Helix Supersoft CSR & $2-4 \mathrm{~mm}$ \\
ev3 & Helix Soft CSR & $5-10 \mathrm{~mm}$ \\
ev3 & MultiDiameter CSR & $2-3 \mathrm{~mm}$ \\
ev3 & Tetris CSR & $3-18 \mathrm{~mm}$ \\
ev3 & Helix Supersoft CSR & $2-5 \mathrm{~mm}$ \\
ev3 & Helix Standard Fiber & $2-3 \mathrm{~mm}$ \\
Micrus & UltiPaq & $2-4 \mathrm{~mm}$ \\
Micrus & Helipaq-10 & $2-6 \mathrm{~mm}$ \\
Micrus & Helipaq-10 & $5-10 \mathrm{~mm}$ \\
Micrus & Helipaq-18 & $2-6 \mathrm{~mm}$ \\
Micrus & Helipaq-18 & $5-20 \mathrm{~mm}$ \\
& & (continued) \\
\hline
\end{tabular}




\begin{tabular}{|c|c|c|}
\hline Company & Type & $D_{3}$ \\
\hline Micrus & MicrusSphere-10 & $2-4 \mathrm{~mm}$ \\
\hline Micrus & MicrusSphere-10 & $5-10 \mathrm{~mm}$ \\
\hline Micrus & MicrusSphere-18 & $2-4 \mathrm{~mm}$ \\
\hline Micrus & MicrusSphere-18 & $5-20 \mathrm{~mm}$ \\
\hline Micrus & Presdio 18 & $8-20 \mathrm{~mm}$ \\
\hline Micrus & Presidio 10 & $5-8 \mathrm{~mm}$ \\
\hline Micrus & Presidio 10 & $4 \mathrm{~mm}$ \\
\hline Micrus & Cashmere 14 & $5-12 \mathrm{~mm}$ \\
\hline Micrus & Cashmere 14 & $2-4 \mathrm{~mm}$ \\
\hline Micrus & Interpaq & $2-10 \mathrm{~mm}$ \\
\hline BSC & GDC-18 STD & \\
\hline BSC & GDC-18 Soft & \\
\hline BSC & GDC-10 STD & \\
\hline BSC & GDC-10 Soft & \\
\hline BSC & GDC-10 UltraSoft & \\
\hline $\mathrm{BSC}$ & GDC-10® 3D Coils & $3-10 \mathrm{~mm}$ \\
\hline Microvention & Helical 10 System & $2-4 \mathrm{~mm}$ \\
\hline Microvention & Helical 10 System & $5-10 \mathrm{~mm}$ \\
\hline Microvention & Helical 18 System & 2-6 mm \\
\hline Microvention & Helical 18 System & $5-20 \mathrm{~mm}$ \\
\hline Microvention & HyperSoft 10 System & $2-6 \mathrm{~mm}$ \\
\hline Microvention & Complex 10 System & $2-3 \mathrm{~mm}$ \\
\hline Microvention & Complex 10 System & $4-10 \mathrm{~mm}$ \\
\hline Microvention & Complex 18 System & $2-4 \mathrm{~mm}$ \\
\hline Microvention & Complex 18 System & $5-7 \mathrm{~mm}$ \\
\hline Microvention & Complex 18 System & 8-12 mm \\
\hline Microvention & Complex 18 System & $13-16 \mathrm{~mm}$ \\
\hline Microvention & Complex 18 System & $18-20 \mathrm{~mm}$ \\
\hline Microvention & Compass 10 System & $2-3.5 \mathrm{~mm}$ \\
\hline Microvention & Compass 10 System & $4-5 \mathrm{~mm}$ \\
\hline Microvention & Compass 10 System & $6-10 \mathrm{~mm}$ \\
\hline Microvention & Compass 18 System & $4-5 \mathrm{~mm}$ \\
\hline Microvention & Compass 18 System & $6-12 \mathrm{~mm}$ \\
\hline Microvention & Compass 18 System & $13-20 \mathrm{~mm}$ \\
\hline
\end{tabular}

\section{Acknowledgments}

The authors wish to thank the following companies for providing information about their coils: Boston Scientific, Cordis, ev3, Microvention, and Micrus.

\section{References}

1. Cloft HJ, Kallmes DF. Aneurysm packing with HydroCoil Embolic System versus platinum coils: initial clinical experience. AJNR Am J Neuroradiol 2004;25:60-62

2. Kawanabe Y, Sadato A, Taki W, et al. Endovascular occlusion of intracranial aneurysms with Guglielmi detachable coils: correlation between coil packing density and coil compaction. Acta Neurochir (Wien) 2001;143:451-55

3. Kimchi TJ, Willinsky RA, Spears J, et al. Endovascular treatment of intracranial aneurysms with Matrix coils: immediate posttreatment results, clinical outcome and follow-up. Neuroradiology 2007;49:223-29. Epub 2007 Jan 3

4. Piotin M, Mandai S, Murphy KJ, et al. Dense packing of cerebral aneurysms: an in vitro study with detachable platinum coils. AJNR Am J Neuroradiol 2000;21:757-60

5. Sluzewski M, van Rooij WJ, Slob MJ, et al. Relation between aneurysm volume, packing, and compaction in 145 cerebral aneurysms treated with coils. Radiology 2004;231:653-58

6. Piotin M, Daghman B, Mounayer C, et al. Ellipsoid approximation versus 3D rotational angiography in the volumetric assessment of intracranial aneurysms. AJNR Am J Neuroradiol 2006;27:839-42

7. Sugahara T, Korogi Y, Nakashima K, et al. Comparison of 2D and 3D digital subtraction angiography in evaluation of intracranial aneurysms. AJNR Am J Neuroradiol 2002;23:1545-52 